REVISTA 89.1 Revista RELACIONES INTERNACIONALES Escuela de Relaciones Internacionales. Universidad Nacional, Costa Rica. N. ${ }^{\circ} 89.1$ • Enero-Junio de 2016 doi: http://dx.doi.org/10.15359/ri.89-1.1

\title{
DISYUNTIVA EN LA NORMATIVA DE ORIGEN ESTABLECIDA EN EL SISTEMA DE PREFERENCIAS GENERALIZADAS DE LA UNIÓN EUROPEA (SPG) Y EL ACUERDO DE ASOCIACIÓN ENTRE CENTROAMÉRICA Y LA UNIÓN EUROPEA (AACUE): CASO COSTA RICA
}

\author{
Christian Cordero Madrigal*
}

\section{RESUMEN:}

La República de Costa Rica cuenta actualmente con dos acuerdos de comercio preferencial con la Unión Europea, el Sistema Generalizado de Preferencias (SPG) y el Acuerdo de Asociación entre Centroamérica y la Unión Europea (AACUE). De forma paralela a la negociación del AACUE, el SPG de la Unión Europea había iniciado un proceso de flexibilización de su normativa de origen, el cual no acabó sino hasta el mismo año en que Centroamérica terminó la negociación del pilar comercial del AACUE. Por tanto, se puede intuir que el tratado más reciente fue negociado sobre la base de una normativa de origen más rígida; lo que implicaría la pérdida de privilegios previamente otorgados por la UE a Costa Rica, al migrar de un esquema de comercio preferencial al otro.

Palabras clave: Normas de origen, acuerdos de comercio preferencial, transformación sustancial, cambio de clasificación arancelaria, valor de contenido regional, proceso productivo, armonización de las normas de origen.

\begin{abstract}
:
The Republic of Costa Rica currently has two preferential trade agreements with the European Union: the Generalized System of Preferences (GSP) and the Association Agreement between Central America and the European Union (EU-CAAA). Similar to the negotiation of the EU-CAAA, the rules of origin with the GSP were flexible until later in the same year, when negotiations with the EU-CAAA ended. Consequently, the most recent treaty was negotiated on the basis of more rigid rules of origin, which implies the loss of privileges that the EU had previously granted Costa Rica when migrating from one trading scheme to another.

Keywords: rules of origin; preferential trade agreements; substantial transformation; change of tariff classification; regional value content; production process; harmonization of rules of origin.
\end{abstract}

\footnotetext{
* Máster en Comercio Exterior, Universidad de Valladolid, España. Correo electrónico: cristian2691@ gmail.com
} 


\section{Introducción}

Existe consciencia de que los acontecimientos económicos que se han presentado durante las últimas décadas, tales como la crisis económica mundial, la aparición de economías emergentes, el aumento de la competencia, la supresión de barreras comerciales y la estandarización de la tecnología mundial, han tenido un profundo impacto en el entorno empresarial. Ello ha obligado a las organizaciones a competir en un mundo turbulento, transformado por el fenómeno de la globalización.

Este hecho trascendental ha dado, como resultado, que las pequeñas y medianas empresas hayan tenido que transformar sus estructuras, estrategias y prácticas gerenciales, aventurándose hacia la internacionalización para poder seguir en el mercado ${ }^{1}$.

De la mano con lo anterior y con la finalidad de mantener una fuerza empresarial competitiva que favorezca el desarrollo económico de un país, los Estados han debido optar por el establecimiento de una serie de acuerdos de comercio preferencial que faciliten la entrada de sus bienes en mercados exteriores.

El comercio preferencial es aquel que refiere a una serie de acuerdos celebrados entre dos o más naciones, donde se conceden condiciones favorables en materia comercial y aduanera, con el afán de liberalizar el comercio entre estas. Este puede ser unidireccional, tal es el caso de los sistemas de preferencias generalizadas en el que un país o región desarrollada otorga condiciones más favorables a un país en vías de desarrollo; o bidireccional, donde el esquema de condiciones favorables se otorga mutuamente, como es el caso de un tratado de libre comercio.

Por otra parte, los firmantes de un acuerdo de comercio preferencial procuran garantizar que únicamente los signatarios sean los favorecidos, evitando desviaciones comerciales a través de terceros países (Corrales, 2011), es decir, solo aquellas mercancías originarias de uno de los países miembros del acuerdo podrá gozar de un tratamiento arancelario preferencial.

Para efectos de una mayor comprensión, el concepto de norma de origen analizado desde el punto de vista multilateral, objeto del presente artículo, adoptará la siguiente definición:

1 Tan solo en el caso de Costa Rica existen 2.470 empresas exportadoras con registros de exportación superiores a 12.000 USD anuales en el año 2013 (728 empresas más que diez años atrás). 


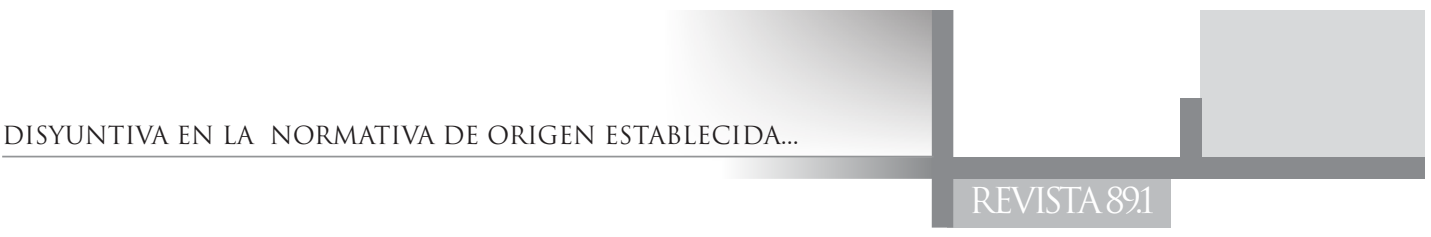

Son los criterios necesarios para determinar la procedencia nacional de un producto. Su importancia se explica porque los derechos y las restricciones aplicados a la importación pueden variar según el origen de los productos importados. (Organización Mundial del Comercio, 2015D)

Pérez \& Grether (1998, p.1) señalan que "el comercio preferencial representó el $40 \%$ del comercio mundial en el período de 1988 a 1992 y aumentó ligeramente hasta el 42\% en el período de 1993 a 1997". Aunado a lo anterior, existen 406 acuerdos comerciales regionales (ACR) ${ }^{2}$ y 27 arreglos comerciales preferenciales (ACPR) ${ }^{3}$ en vigor, notificados ante la Organización Mundial del Comercio (OMC) por sus miembros (Organización Mundial del Comercio, 2015B).

Como se pone de manifiesto, gran parte del comercio mundial se encuentra al margen de acuerdos preferenciales. Una vez vigente el esquema preferencial pactado, es necesario el cumplimiento de una serie de normas para lograr el acceso a determinados mercados; en ese sentido, la determinación del origen de una mercancía es uno de los requisitos necesarios para gozar de un tratamiento arancelario preferente. Sin embargo, muchas veces estas normas establecen condiciones cargadas de rigidez que han de ser cumplidas por las mercancías para ser consideradas originarias y, en el esquema comercial actual, donde existen muy pocos productos en los que todos los insumos utilizados provengan de un único país, resulta bastante complejo para los productores cumplir con estas condiciones. Por ello, no les queda otra alternativa que confiar en los creadores de política comercial de sus respectivos países para que, al negociar un acuerdo de comercio preferencial, logren concretar reglas de origen, lo suficientemente flexibles, que les permitan beneficiarse de los mismos acuerdos.

Por citar un ejemplo que se apega directamente al tema de este artículo, en el caso específico del Sistema de Preferencias Generalizadas (SPG) de la Unión Europea, Brenton \& Manchin (2002), en su estudio titulado Making EU Trade Agreements Work: The Role of Rules of Origin, han calculado el porcentaje de aprovechamiento del mismo sistema por parte de los países beneficiarios y señalan que, a pesar de que, en el año 1999, el 99\% de las exportaciones a la UE realizadas por los signatarios fueron con el amparo del Acuerdo, tan solo un $31 \%$ de las exportaciones lograron entrar al mercado europeo bajo un esquema de aranceles preferenciales. Los autores aluden a que el principal motivo del

2 Los acuerdos comerciales regionales (ACR) se definen como acuerdos comerciales recíprocos entre dos o más socios. Incluyen los acuerdos de libre comercio y las uniones aduaneras (Organización Mundial del Comercio, 2015A).

3 Los arreglos comerciales preferenciales (ACPR) son, en la OMC, preferencias comerciales unilaterales. Incluyen los esquemas adoptados en el marco del Sistema Generalizado de Preferencias en virtud del cual los países desarrollados aplican aranceles preferenciales a las importaciones procedentes de los países en desarrollo (Organización Mundial del Comercio, 2015A). 
bajo nivel de aprovechamiento eran las reglas de origen restrictivas impuestas por la UE, aunado a los costos de su cumplimiento. Esta situación evidencia la importancia del presente estudio.

De esta manera, se considera que "las reglas de origen deberán considerarse instrumentos de política comercial primarios en toda negociación de acceso a los mercados, y no solo auxiliares en la aplicación de un instrumento primario como son los aranceles preferenciales" (Estevadeordal, 2003, p. 9).

Por todo lo anterior, el presente artículo analiza, de manera comparativa, la normativa de origen establecida en dos acuerdos de preferencias arancelarias hasta ahora vigentes en Costa Rica: el Acuerdo de Preferencias Generalizadas (SPG), como esquema de preferencias unidireccional ${ }^{4}$ que otorga la Unión Europea a los países en desarrollo; y el Acuerdo de Asociación entre Centroamérica y la Unión Europea (AACUE), como un acuerdo bidireccional en el que ambas regiones se conceden preferencias comerciales; esto, con la pretensión de determinar las posibles disyuntivas que, en materia de origen, se generan y que podrían afectar el entorno empresarial costarricense, una vez que Costa Rica se retire del listado de beneficiarios del SPG, en el año 2016. Este hecho podría repercutir en el acceso preferencial de sus mercancías a la Unión Europea y, por tanto, no cabe duda de la importancia de la coexistencia de ambos acuerdos para el país.

\section{Antecedentes}

Como se ha manifestado con anterioridad, el origen de las mercancías es un elemento primordial para que los países puedan gozar de tasas arancelarias reducidas o nulas al margen de sus acuerdos comerciales preferenciales.

La coexistencia actual de los dos acuerdos comerciales para Costa Rica es la consecuencia de sendos procesos de negociación que se han llevado a cabo de forma paralela, los cuales se explican a continuación (ver figura 1).

\section{a) Proceso de flexibilización del SPG}

Debido a su importancia, en el año 2003 se inicia un debate en la Unión Europea, conocido como el "Libro verde", en el que se discute sobre el futuro de

4 Preferencias en una sola dirección, es decir, otorgadas por la UE a los países en desarrollo sin esperar que estos países concedan preferencias similares a cambio.

5 Véase la metodología utilizada en la sección 3 del presente artículo. 


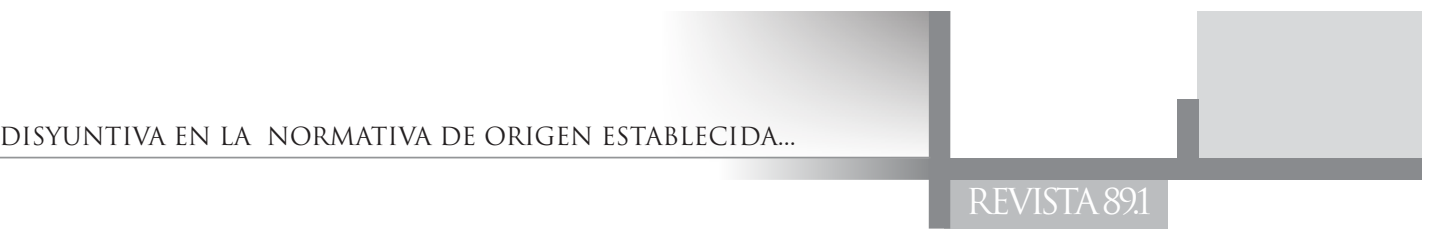

las normas de origen en los regímenes comerciales preferenciales; lo anterior, en virtud de las condiciones de acceso a través de aranceles preferentes que la Unión Europea otorga a sus socios comerciales. En dicho debate se toma consciencia de que estos regímenes comerciales solo tienen sentido, si las preferencias arancelarias benefician a las mercancías que han sido obtenidas de manera efectiva en el territorio de una de las partes del acuerdo, es decir, si las mercancías cumplen con la regla de origen pactada y pueden considerarse originarias.

Respecto a la normativa de origen establecida en estos acuerdos preferenciales, la Comisión de las Comunidades Europeas (2003), en su debate Libro verde: El futuro de las normas de origen en los acuerdos comerciales preferenciales menciona:

El marco actual para determinar, gestionar y controlar el origen preferencial ya no está adaptado plenamente ni a estas necesidades, ni a la evolución cuantitativa y cualitativa de la economía internacional. Esta evolución impone, en particular, trascendiendo una búsqueda de la aplicación correcta de las normas de origen, la necesidad de evaluar de nuevo su pertinencia económica, mejorar la fiabilidad del sistema para proteger los intereses financieros de la Comunidad y restablecer una división equilibrada de las responsabilidades entre los operadores beneficiarios de las preferencias y las autoridades encargadas de los controles. (Comisión de las Comunidades Europeas , 2003)

Es decir, la Comisión Europea empieza a tomar consciencia de que la normativa de origen pactada en sus acuerdos comerciales preferenciales se encuentra cargada de rigidez, por lo que resulta bastante complejo, para los beneficiarios de los acuerdos, acceder al mercado europeo bajo un esquema de aranceles preferentes. En ese sentido, el Libro verde pretende simplificar y hacer menos rigurosas las reglas de origen y mejorar el aprovechamiento de las preferencias concedidas a los países favorecidos. De esta manera, el 18 de noviembre de 2010 se establece una flexibilización de la normativa de origen del Acuerdo Generalizado de Preferencias ${ }^{6}$ del que Costa Rica es beneficiaria.

La nueva normativa de origen del SPG presenta cambios tanto en las reglas específicas de origen, como en aspectos relativos a los artículos en lo que refiere a los criterios para determinar el origen, la acumulación de origen y, así mismo, se establece un nuevo sistema de exportador autorizado.

6 A través del Reglamento (UE) No 1063/2010 de la Comisión Europea. 


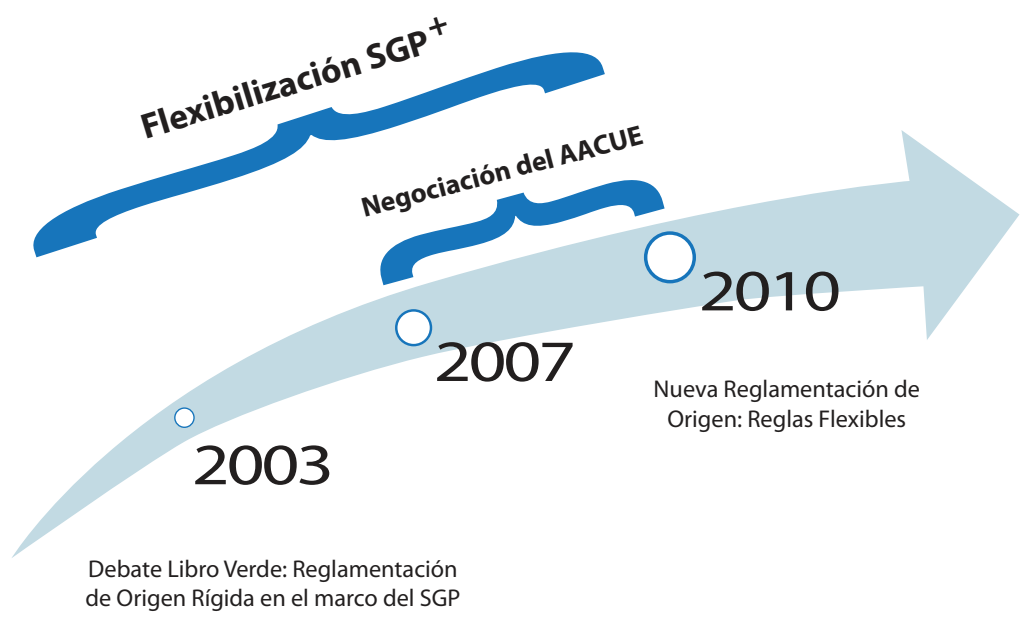

Figura 1. La disyuntiva en materia de origen entre el SPG y el AACUE. Elaboración propia.

En cuanto a los criterios para determinar el origen de una mercancía, específicamente el criterio de transformación sustancial, es decir, cuando una mercancía incorpora insumos no originarios, se toma consciencia de que el principio de valor de contenido regional resultaba sumamente restrictivo y, por tanto, no debía ser utilizado en el sector de productos agrícolas, pesca, químicos, metales, confección y calzado (Martínez Jerez, 2011). El principio de valor de contenido regional era una constante, pero para los sectores mencionados es sustituido por criterios como el cambio de clasificación arancelaria, de mínimis o contenido máximo de insumos no originarios, es decir, se mantiene un enfoque sectorial y no por mercancías.

En cuanto a la regla de tolerancia (de mínimis) se incrementa el umbral de contenido de insumos no originarios pasando de un $10 \%$ a un $15 \%$.

Se permite la acumulación bilateral, la regional y se establece un nuevo régimen de acumulación denominado acumulación ampliada.

En lo que refiere a la acumulación bilateral, en la que los insumos producidos en países que conforman la UE serán considerados originarios del país beneficiario, se mantiene la posibilidad de acumular origen ${ }^{7}$ con países como Noruega y Suiza, pero este criterio se extiende a Turquía en el nuevo reglamento.

7 Aplica para productos industriales. 
Por su parte, la acumulación regional se establece a partir de 4 grupos regiona$\operatorname{les}^{8}$, siendo el grupo IV un nuevo grupo de acumulación regional que incorpora el reglamento:

- Grupo I: Brunéi Darusalam, Camboya, Indonesia, Filipinas, Laos, Malasia y Vietnam.

- Grupo II: Bolivia, Colombia, Costa Rica, El Salvador, Guatemala, Honduras, Nicaragua, Panamá, Perú y Venezuela.

- Grupo III: Bangladés, Bután, India, Nepal, Pakistán y Sri Lanka

- Grupo IV: Argentina, Brasil, Paraguay y Uruguay

- Por otra parte, el nuevo reglamento permite la acumulación de origen entre los países de los grupos I y III descritos anteriormente.

- En cuanto a la acumulación ampliada como un nuevo esquema de acumulación, se permite considerar, como propios, los insumos provenientes de países con los que la Unión Europea mantenga un Acuerdo de Libre Comercio?.

Otro de los cambios que maneja el nuevo reglamento de origen del SPG es que, a partir del 2017, los certificados de origen ${ }^{10}$ pasarán a la historia y serán sustituidos por un sistema de exportadores registrados en el seno de este nuevo reglamento. Será responsabilidad de los países beneficiarios del Acuerdo crear el registro electrónico de exportadores y mantenerlo constantemente actualizado; de la misma manera, los organismos correspondientes del país beneficiario deberán comunicar el registro a la Comisión Europea, la cual se encargará de establecer una base de datos centralizada.

\section{b) Proceso de negociación del AACUE}

En mayo de 2006, en el marco de la VI cumbre Unión Europea-América Latina y el Caribe, celebrada en Viena, los representantes ejecutivos de los bloques europeo y centroamericano decidieron dar inicio a un proceso de negociación,

8 La acumulación regional se permite entre países del mismo grupo.

9 Este tipo de acumulación es unidireccional, es decir, únicamente los beneficiarios del SPG podrán acumular origen con los países con los que la UE mantenga un Acuerdo de Libre Comercio y no en viceversa.

10 Certificados de Origen Modelo A 
cuyo objetivo era la celebración del AACUE, estableciendo compromisos en tres grandes áreas: diálogo político, cooperación y comercio.

Después de una serie de rondas de negociación, el proceso culmina tras la ronda de Madrid, efectuada del 12 al 18 de mayo de 2010 (Ministerio de Comercio Exterior de Costa Rica, 2012). El pilar comercial de este Acuerdo entró en vigor para Costa Rica a partir del 1 de octubre del 2013.

Por tanto, el proceso de negociación del AACUE culminó en Madrid en el año 2010; sin embargo, el Sistema de Preferencias Generalizadas, como esquema de acceso preferencial al mercado europeo para Costa Rica, inició un proceso de debate en pro de la flexibilización de la normativa de origen desde el año 2003 y no concluyó sino hasta el 2010 (año en que las negociaciones del AACUE culminaron). Por todo ello se puede inferir que Centroamérica negoció un nuevo Acuerdo con la Unión Europea sobre la base de normas de origen más rígidas (ya que la normativa de origen flexibilizada del SPG no fue publicada hasta el 2010) y, por tanto, cabe la posibilidad de que la nueva reglamentación de origen del SPG conserve normas más flexibles que el actual esquema de preferencias entre Centroamérica y la Unión Europea (AACUE).

\section{c) Conclusión de la coexistencia del SPG y el AACUE}

El Reglamento (CE) N.o 732/2008 del Consejo Europeo, por el que se aplica un sistema de preferencias generalizadas vigente hasta el 31 de diciembre de 2013, en su artículo 3, párrafo 2, establece:

Cuando un país beneficiario haya suscrito un acuerdo preferencial con la Comunidad que abarque todas las preferencias establecidas para él, en el presente sistema, se retirará de la lista de países beneficiarios. (Consejo de la Unión Euopea, 2008)

Sin embargo, el 1 de enero de 2014 empezó a aplicarse, en la Unión Europea, un nuevo Sistema de Preferencias Generalizadas ${ }^{11}$ y Costa Rica sigue siendo parte de este Sistema, es decir, los Acuerdos coexisten; pero el centroamericano se encuentra ante un periodo de transición desde el 1 de octubre de 2013 y dejará de ser beneficiario del SPG a partir del 1 de enero del 2016, lo que puede ser comprobado a partir del Reglamento Delegado UE N.o 1015/2014, del 22 de julio del 2014, donde textualmente se expresa:

11 Reglamento (UE) No 978/2012 
Con los siguientes países comenzaron a aplicarse acuerdos de acceso preferencial al mercado en diversos momentos de 2013: Perú, el 1 de marzo; Colombia, Honduras, Nicaragua y Panamá, el 1 de agosto; Costa Rica y El Salvador, el 1 de octubre; y Guatemala, el 1 de diciembre. Con el fin de garantizar una aplicación uniforme del cambio producido en el estatus del país dentro del SPG, y en consonancia con el Reglamento SPG, Perú, Colombia, Honduras, Nicaragua, Panamá, Costa Rica, El Salvador y Guatemala deben suprimirse del anexo II $^{12}$ a partir del 1 de enero de 2016.

En el caso específico de Costa Rica, al ser parte tanto del Régimen General ${ }^{13}$ del SPG, como del Régimen Especial ${ }^{14}$ de Estímulo al desarrollo sostenible y la Gobernanza ( $\mathrm{SPG}+)$, el retiro contemplará ambos regímenes de acceso preferencial, tal como lo describe el reglamento mencionado:

Al dejar de ser beneficiarios del SPG, Costa Rica, Guatemala, Perú, Panamá y El Salvador también dejan de serlo del SPG+, de conformidad con el artículo 9, apartado 1, del Reglamento SPG. Así, estos países deben suprimirse del anexo III del Reglamento SPG a partir del 1 de enero de 2016. (Comisión Europea, 2014)

Tras la disyuntiva en cuanto a tiempo y normativa base para la negociación del AACUE, nace la inquietud por conocer y darle respuesta al problema implícito en la presente pregunta: ¿Existen algunas normas de origen más rígidas en el AACUE que en el SPG y, por tanto, qué pasará una vez que Costa Rica sea retirado de la lista de beneficiarios del último, en enero de 2016 ?

\section{Metodología}

La fuente de información utilizada es denominada análisis de contenido; en cuanto a este tipo de estudios, Piñuel Raigada (2002, p. 2) manifiesta:

12 En el anexo II del Reglamento SPG se establece la lista de países beneficiarios del régimen general del SPG

13 Ofrece un trato preferencial básico a los países beneficiarios. En el caso de los productos considerados como no sensibles este régimen ofrece exención arancelaria, mientras que en el caso de los productos sensibles existe una reducción arancelaria de 3,5 puntos porcentuales en relación con el arancel NMF y en el caso específico de los textiles y las prendas de vestir la reducción es del 20 por ciento.

14 Este régimen ofrece un estímulo al desarrollo sostenible y la gobernanza, otorgando beneficios arancelarios adicionales (en el caso de las mercancías sensibles ya que las no sensibles son exentas en el régimen general) a aquellos países en desarrollo que ratifiquen y apliquen adecuadamente Convenciones Internacionales en materia de derechos humanos, laborales de medio ambiente y buen gobierno. 
Se suele llamar análisis de contenido al conjunto de procedimientos interpretativos de productos comunicativos (mensajes, textos o discursos) que proceden de procesos singulares de comunicación previamente registrados, y que, basados en técnicas de medida, a veces cuantitativas (estadísticas basadas en el recuento de unidades), a veces cualitativas (lógicas basadas en la combinación de categorías), tienen por objeto elaborar y procesar datos relevantes sobre las condiciones mismas en que se han producido aquellos textos, o sobre las condiciones que puedan darse para su empleo posterior.

El análisis estará basado en el contenido de las reglas de origen específicas establecidas en dos acuerdos de comercio preferencial de los que la República de Costa Rica es beneficiaria; concretamente se analizará el contenido del Reglamento (UE) N.o 1063/2010 mediante el cual se establece una nueva normativa de origen, al amparo del Sistema de Preferencias Generalizadas de la Unión Europea y el Anexo II (relativo a la definición del concepto de productos originarios) del Acuerdo de Asociación entre Centroamérica y la Unión Europea.

El estudio se basa en una técnica cuantitativa a través de una matriz comparativa en la que se analizan, conjuntamente, 662 reglas de origen de cada Acuerdo y en la que se pretende determinar el porcentaje de reglas de origen más rígidas que posee el AACUE, en relación con el SPG. Es preciso tener en cuenta que el SPG dejará de estar en vigor para Costa Rica a partir del 16 de enero del 2016.

Las reglas de origen de cada Acuerdo se encuentran segmentadas en relación con la nomenclatura del Sistema Armonizado de Designación y Codificación de Mercancías y comprende reglas de origen para los diferentes capítulos, partidas y subpartidas del mismo. Es necesario indicar que para efectos del presente estudio fueron descartados los capítulos 50 a $63^{15}$ de la nomenclatura por dos razones: la industria textil es una industria incipiente para Costa Rica que ha dedicado sus esfuerzos a la participación en el mercado de media y/o alta tecnología y además las reglas de origen están cargadas de tecnisismos propios de esta, por lo que para su interpretación es necesario conocer a profundidad este sector.

Con el objeto de interpretar las reglas de origen serán considerados cuatro criterios (ver figura 2).

15 Estos capítulos comprenden las materias textiles y sus manufacturas 


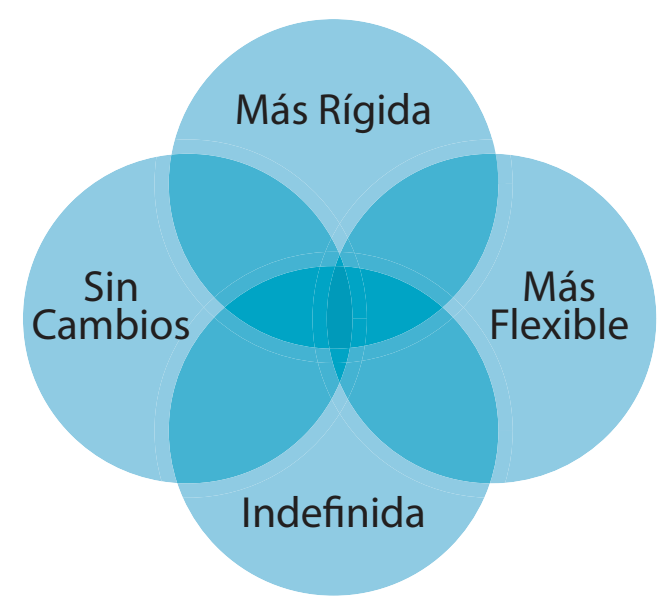

Figura 2. Criterios para la interpretación de las reglas de origen. Elaboración propia.

\section{Definición del concepto de regla de origen más rígida}

El concepto de regla de origen más rígida se podría definir, en términos generales, como aquella regla de origen que se aleja del criterio de transformación sustancial para apegarse al esquema del criterio de obtención total o totalmente obtenido, donde la importación de insumos foráneos hace perder el origen de la mercancía a la que alude la regla.

Sin embargo, la definición anterior resulta muy escueta y universal, diferenciando únicamente entre los criterios de obtención total y de transformación sustancial. No obstante, para determinar reglas de origen más rígidas es necesario acudir al criterio de origen específico al que la regla alude. En ese sentido, se establecerá una definición de regla de origen más rígida basada en los subcriterios que conforman la transformación sustancial; todo esto desde la aseveración de que una regla de obtención total será siempre más rígida.

Resulta importante adelantar que cuando se alude a la transformación sustancial, no podría generalizarse qué tipo de regla es más restrictiva que otra en el caso de que ambos acuerdos apunten a dos criterios distintos de determinación de origen para un mismo producto o conjunto de ellos, sino que cada caso debe ser analizado en particular. Por otra parte, se podrían establecer razonamientos uniformes respecto a la rigidez de la norma, cuando un producto o grupo de 
ellos estén representados por reglas de origen que obedezcan a un mismo criterio de determinación de origen; a saber, cambio de clasificación arancelaria, valor de contenido regional o proceso productivo. Estas consideraciones serán ampliadas a continuación:

\section{a) Cambio de clasificación}

Se considera que un producto ha sido transformado sustancialmente si la clasificación arancelaria del producto final cambia respecto a la clasificación arancelaria de las materias primas o insumos importados de países que no son parte del acuerdo. Dicho de otra manera y a modo de ejemplo, las fresas frescas dejan de serlo en virtud del procesamiento al que han sido sometidas para convertirse en una mermelada.

El grado de restricción de una regla de origen basada en el criterio de cambio de clasificación arancelaria se encuentra determinado por la magnitud del salto arancelario solicitado (ver figura 3).

Por tanto, una regla de origen con cambio de clasificación arancelaria a nivel de partida es más restrictiva que a nivel de subpartida; asímismo, un cambio de clasificación a nivel de capítulo restringe más que un cambio a nivel de partida, por lo que se puede inferir que un cambio de clasificación arancelaria a nivel de capítulo es siempre más restrictivo.

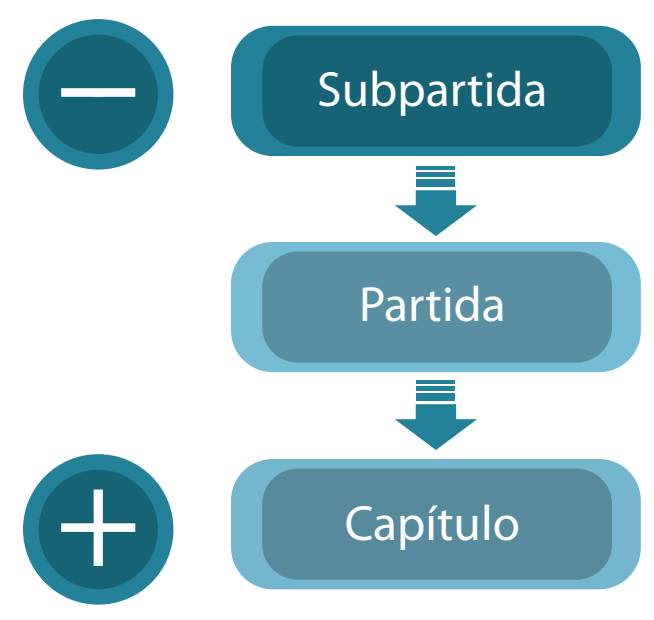

Figura 3. Nivel de restricción del cambio de clasificación arancelaria. Elaboración propia. 


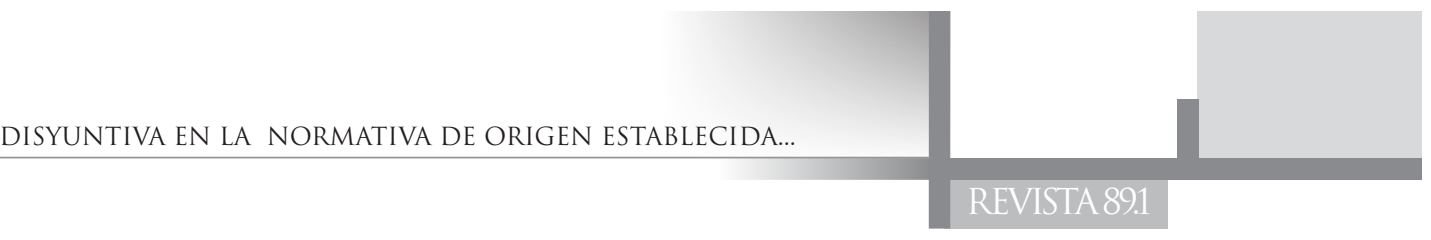

De la misma manera, una regla de origen, basada en este criterio, es más restrictiva en cuanto se exceptúe la importación desde terceros países de mayor cantidad de mercancías o insumos clasificados en las diferentes subpartidas, partidas o capítulos de la nomenclatura del Sistema Armonizado.

\section{b) Valor de contenido regional}

El criterio de valor de contenido regional también es conocido como porcentajes ad valorem, criterio económico o método del valor añadido. Respecto de este criterio, López Libreros $(2008$, p. 169) manifiesta que "se establecen porcentajes de valores máximos y mínimos que los procesos de transformación de materiales e insumos no originarios deben suponer respecto del producto final". Es decir, este puede ser expresado de dos maneras: se establecen porcentajes máximos de insumos no originarios que pueden incorporar un bien final o, dicho de otra manera, porcentajes mínimos de insumos originarios con los que debe contar el bien terminado para ser considerado originario.

Una regla de origen basada en el criterio de valor de contenido regional será más restrictiva cuanto mayor sea el porcentaje de insumos originarios solicitado.

\section{c) Proceso productivo}

Este criterio también es conocido como operación de fabricación o elaboración o procesos técnicos y refiere a las transformaciones o procesos a los que deben ser sometidos los insumos para que otorguen origen al producto final.

Las reglas de origen más rígidas en cuanto a este criterio serán aquellas que exijan procesos productivos más sofisticados que impliquen la puesta al día, conforme al avance de los procesos productivos.

\section{Otros criterios empleados}

Para completar el análisis, además de establecer la regla más rígida y su opuesta, la más flexible, se recurrirá a otros razonamientos:

\section{a) Indefinido}

Se considerará que existe indefinición de dos reglas de origen cuando no es posible determinar cuál de ellas es más rígida o más flexible y esto sucede debido a que ciertas reglas de origen obedecen a juicios cargados de relatividad, donde es necesario conocer el esquema productivo de cada exportador para 
poder determinar si la regla es más rígida o más flexible. Por tanto, las reglas de origen catalogadas como indefinidas pueden resultar más rígidas para ciertos empresarios, en tanto que para otros pueden ser más flexibles; todo ello dependerá de su esquema de producción.

Adicionalmente, las reglas de origen serán consideradas indefinidas cuando establezcan porcentajes mínimos o máximos; pero estos porcentajes son calculados sobre la base de dos términos de medición distintos (tabla 1).

Tabla 1.

Indefinición de una regla de origen

\begin{tabular}{|l|l|}
\hline \multicolumn{1}{|c|}{ Designación } & \multicolumn{1}{|c|}{ Regla } \\
\hline \multirow{2}{*}{$\begin{array}{l}\text { Frutas y frutos } \\
\text { comestiblesmelones } \\
\text { o sandías }\end{array}$} & $\begin{array}{l}\text { El peso del azúcar utilizado no exceda del } 40 \% \text { del peso del } \\
\text { producto acabado (SPG). }\end{array}$ \\
\cline { 2 - 2 } & $\begin{array}{l}\text { El valor de todos los materiales del capítulo } 17 \text { utilizados no ex- } \\
\text { ceda del 30\% del precio franco fábrica del producto (AACUE). }\end{array}$ \\
\hline
\end{tabular}

Fuente: Elaboración propia.

\section{b) Sin cambios}

Existen reglas de origen que no representan ningún cambio de un Acuerdo a otro más que cuestiones mismas de redacción y, por tanto, no representan ninguna amenaza para el beneficiario del acuerdo.

\section{Resultados}

Los resultados obtenidos tras el análisis comparativo de las 662 reglas de origen de cada Acuerdo, donde se obedece a los criterios de mayor rigidez, mayor flexibilidad, indefinición de la comparativa o las reglas de origen en las que no se presentaron cambios, demuestran que tal como se planteó al inicio del presente estudio, el nuevo esquema de preferencias arancelarias entre Centroamérica y la Unión Europea (AACUE) posee reglas de origen más restrictivas que su 
homólogo el Sistema de Preferencias Generalizadas de la Unión Europea (ver figura 4). Resultados

De un total de 662 reglas de origen comparadas, el AACUE contiene 257 reglas cargadas de una rigidez mayor que representan $38,8 \%$ del total.

Adicionalmente, tan solo un 9,9\% (66) de las reglas son más flexibles, lo que deja como resultado un saldo neto de $28,9 \%$ de mayor rigidez en las reglas de origen específicas del nuevo Acuerdo.

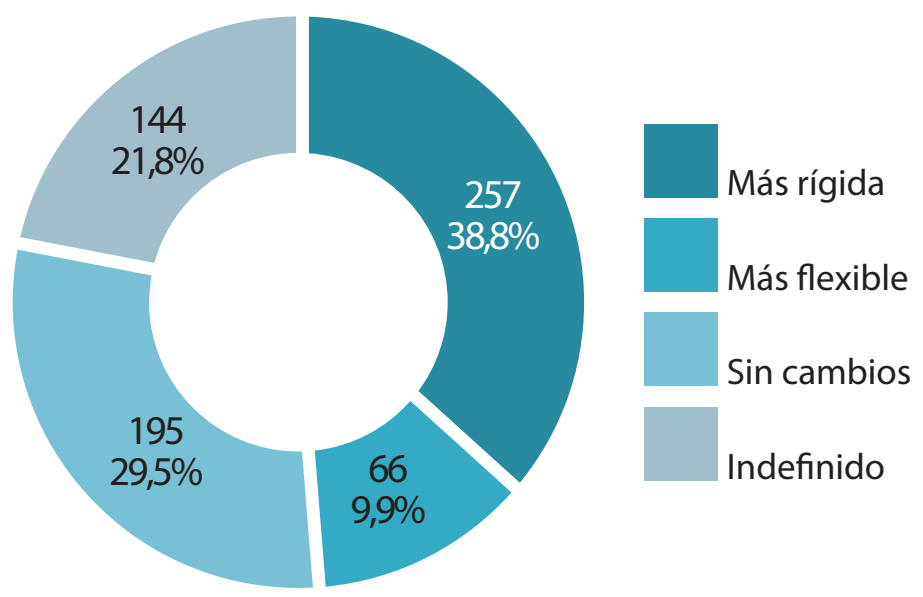

Figura 4. Análisis de las reglas de origen del AACUE en relación con el SPG. Elaboración propia.

Por otra parte, tras el diagnóstico realizado fue imposible determinar la condición de 144 reglas, por lo que $21,8 \%$ de ellas fue categorizado como indefinido y, además, un 29,5\% (195 reglas) no presentó cambios en el AACUE, respecto a sus iguales en el SPG.

La presencia de 247 reglas de origen más rígidas en el AACUE podría suponer un verdadero problema para los exportadores costarricenses, quienes gozaban de los privilegios de acceso al mercado de la UE para colocar sus mercancías a través de un sistema más flexible como es el SPG. Migrar al AACUE supondría la imposibilidad de beneficiarse del trato arancelario preferencial, debido al incumplimiento de la normativa de origen o; en otra instancia, implicaría la necesidad de readaptar su estructura productiva para cumplir con esta normativa, lo que se podría convertirse en mayores costos y, por tanto, en una posible pérdida de competitividad de sus productos. 
Los diferentes sectores productivos de un país depositan su confianza en los creadores de política comercial y hacen escuchar su voz respecto a sus necesidades; mas resulta un desliz, en el juego de poder presente en cualquier negociación comercial, que las delegaciones centroamericanas hayan negociado un nuevo Acuerdo en el que se deba renunciar a beneficios ya otorgados por la UE, con otro esquema preferencial. Por el contrario, el afán de dichas autoridades debería ser buscar mejores resultados a los ya obtenidos o, al menos, mantenerlos.

Independientemente de que este estudio se limite tan solo a uno de los capítulos del Acuerdo, resulta preocupante el hecho de que migrar a un nuevo sistema implique la pérdida de privilegios anteriormente concedidos. Este debate se intensificará a partir de enero de 2016, cuando Costa Rica sea retirada de la lista de beneficiarios del SPG y los productores tengan conciencia de las consecuencias del cambio.

Precisamente, otro de los inconvenientes más alarmantes producto de los resultados obtenidos en este artículo es que, una vez que Costa Rica sea retirado de la lista de beneficiarios del SPG, los países que permanezcan en el régimen podrían tener mejores condiciones de acceso al mercado europeo que las que tendría Costa Rica vía AACUE, tal es el caso de países como: Brunéi, Darusalam, Camboya, Indonesia, Filipinas, Laos, Malasia, Vietnam, Bolivia, Venezuela, Bangladés, Bután, India, Nepal, Pakistán, Sri Lanka, Argentina, Brasil, Paraguay y Uruguay.

No puede obviarse que se pasa de un esquema de preferencias unidireccionales a un esquema de preferencias en doble vía, es decir, con el SPG resultaba siempre conveniente tener reglas de origen más flexibles, ya que Costa Rica podría beneficiarse de esto sin retribuir a cambio los mismos beneficios a la UE. Sin embargo, al amparo del AACUE no sucede lo mismo, por su carácter bidireccional. Es decir, no siempre resulta más beneficioso tener reglas de origen más flexibles, pues estas interesan a los sectores más competitivos, en tanto que sectores sensibles han de ser protegidos ante la entrada de un gigante, como lo es la UE, y las reglas de origen más estrictas o los aranceles elevados podrían ser medios para lograrlo. Por tanto, las normas han de ser evaluadas de la mano del sector al que se aplican.

Desde la perspectiva anterior, la matriz comparativa en la que se evalúan las reglas de origen del AACUE, en relación con el SPG, permite valorar las reglas de origen específicas para los sectores más competitivos de Costa Rica en relación con la UE, introduciendo los principales productos de exportación del centroamericano en el año 2013, específicamente al territorio que conforma la Unión Europea. 


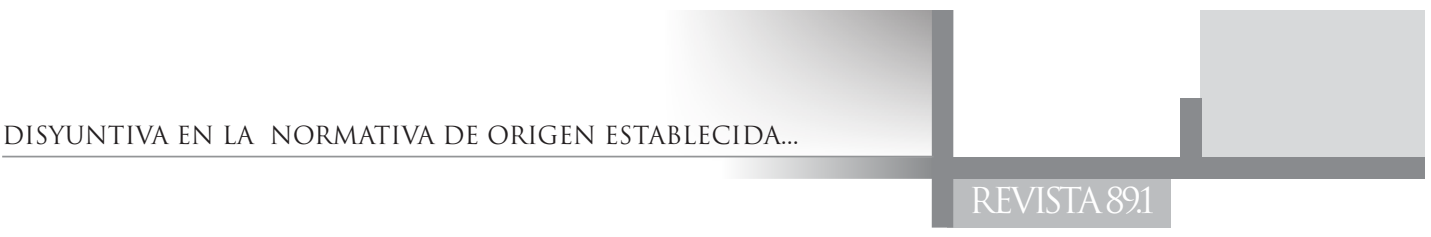

En el caso de algunos de estos productos, ser catalogados como competitivos y con amplia aceptación en el mercado europeo les beneficia en la apertura comercial y, por tanto, en reglas de origen más flexibles que les permitan entrar a este mercado con mayor facilidad; en otros casos es conveniente una regla más rígida.

De los 14 principales productos de exportación de Costa Rica a la Unión Europea, la mitad de ellos tiene reglas de origen más rígidas en el AACUE, en tanto que ninguno goza de reglas de origen más flexibles que las establecidas en el SPG. Por su parte, dos permanecen sin cambios entre los Acuerdos; mientras que en cinco de las reglas no se pudo establecer comparabilidad.

Para ejemplificar lo anterior, se recurrirá al principal producto de exportación de Costa Rica a la Unión Europea: los circuitos integrados, que en el año 2013 representaron 397,5 millones de dólares. En Costa Rica la empresa Intel ${ }^{16}$ posee una planta de ensamblaje y prueba de estas mercancías; por tanto, es conveniente la presencia de una regla de origen flexible que permita la importación de insumos de terceros países. Cuanto mayor sea la cantidad de insumos de importación permitidos, mayor será la flexibilidad de la regla y la facilidad de Intel Costa Rica para cumplirla. De esta manera, podrá beneficiarse del trato arancelario preferencial y seguir siendo competitiva en la UE. En el caso contrario, cuanto más rígida sea la regla de origen, Intel Costa Rica no podrá cumplirla o, en el mejor de los casos, deberá modificar su estructura productiva seleccionando nuevos proveedores, lo que podría restarle competitividad. Sin embargo, el AACUE presenta una regla de origen más rígida para los circuitos integrados que su similar el SPG de la Unión Europea, lo que constata que el nuevo acuerdo genera una gran disyuntiva en materia de origen de las mercancías.

Al igual que en el ejemplo anterior, algunos de los 14 principales productos de exportación (productos competitivos) precisan de reglas más flexibles en el nuevo Acuerdo o al menos que no existan cambios para no perder los privilegios ya obtenidos en el SPG. Esta es la situación de las prótesis de uso médico, los equipos de infusión y transfusión de suero, las agujas, catéteres o cánulas; sin embargo, esto no sucede (ver tabla 2).

En cuanto a los productos agrícolas como la piña o el banano, incluso los jugos de frutas, resultan convenientes reglas de origen más rígidas, donde no se permita la importación de la fruta de terceros países para desarrollar productos a partir de ellos; sin embargo, esto no sucede en el AACUE.

16 Empresa multinacional fabricante de circuitos integrados y microprocesadores especializados y principal representante de las exportaciones costarricenses del sector, genera alrededor del 98\% exportado anualmente. 
Únicamente en el café se puede apreciar una ventaja por parte del nuevo acuerdo a través de una regla de origen más rígida ${ }^{17}$.

Tabla 2.

Condición de las reglas de origen de los principales productos de exportación de Costa Rica a la Unión Europea en el año 2013

\begin{tabular}{|l|c|c|}
\hline \multicolumn{1}{|c|}{ Mercancía } & Clasificación & Condición AACUE \\
\hline Circuitos Integrados & 8542 & Más rígida \\
\hline Piña & 0803 & Indefinido \\
\hline Banano & 0803 & Indefinido \\
\hline Café & 0901 & Más rígida \\
\hline Jugos de frutas & 2009 & Indefinido \\
\hline Prótesis de uso médico & 9021 & Más rígida \\
\hline $\begin{array}{l}\text { Equipos de infusión } \\
\text { y transfusión de suero }\end{array}$ & 9018 & Más rígida \\
\hline Follajes & 0604 & Sin cambios \\
\hline Azúcar & 1701 & Más rígida \\
\hline Melón & 0807 & Indefinido \\
\hline Plantas ornamentales & 0604 & Sin cambios \\
\hline Alcohol etílico & 2207 & Más rígida \\
\hline Purés y pastas de frutas & 2007 & Indefinido \\
\hline Agujas, catéteres y cánulas & 9018 & Más rígida \\
\hline
\end{tabular}

Fuente: Elaboración propia.

Este resultado demuestra, una vez más, las incongruencias que presenta el AACUE en relación con el SPG en cuanto a las reglas de origen específicas. Lo anterior como resultado de un proceso de negociación sobre la base de una normativa de origen más rígida del Sistema de Preferencias Generalizadas que, de forma paralela a la negociación del AACUE, fue experimentando un proceso de flexibilización no concluido sino hasta el mismo año en que terminaron las negociaciones del pilar comercial del AACUE.

Si bien es cierto, resulta necesario analizar caso por caso $^{18}$ para determinar si las nuevas reglas del AACUE resultan inconvenientes para los productores

17 Esto al margen del análisis de los 14 principales productos de exportación.

18 Interpretación de la norma al margen del sector y del producto específico. 
costarricenses, lo que sí es un hecho es que los países que seguirán siendo beneficiarios del SPG tendrán mejores condiciones de acceso al mercado de la UE en relación con Costa Rica y demás países centroamericanos. Esto se debe a que se enfrentarán a un $38,8 \%$ de reglas de origen más rígidas al amparo del AACUE.

Por tanto, la labor de los creadores de política comercial de Costa Rica, específicamente del Ministerio de Comercio Exterior, no fue concretada exitosamente desde el punto de vista de las normas de origen, sino que por el contrario, migrar al AACUE supondría dificultades para algunos sectores productivos del país, sectores que ya gozaban de privilegios con el SPG.

\section{Conclusiones}

Las normas de origen en los acuerdos comerciales preferenciales se convierten en una especie de cancerberos; ya que, por una parte, permiten el acceso a las preferencias pactadas por los signatarios; pero, por otra, restringen el acceso a terceros. Sin embargo, el otorgamiento de una preferencia en el marco de un acuerdo de comercio preferencial no tendrá sentido, si el costo del cumplimiento de la norma de origen para los exportadores es muy alto.

Una norma de origen cargada de rigidez podría ocasionar distorsión comercial y pérdida de la competitividad para el productor o exportador, pues, con el afán de acceder al tratamiento arancelario preferencial, estos pueden verse obligados a renunciar a insumos más eficientes provenientes de terceros países para cumplir con la regla de origen específica.

La República de Costa Rica es actualmente beneficiaria de dos acuerdos de comercio preferencial con la Unión Europea: el primero de ellos, el Sistema de Preferencias Generalizadas de la Unión Europea, es un acuerdo unidireccional; mientras que el segundo, denominado Acuerdo de Asociación entre Centroamérica y la Unión Europea, de reciente vigencia, tiene un carácter bidireccional o regional. De forma paralela a la negociación del AACUE, la normativa de origen del SPG experimentó un proceso de flexibilización que concluyó en una nueva normativa, dada a conocer en el año 2010, cuando Centroamérica concluyó el proceso de negociación del AACUE.

Por lo tanto, la normativa de origen del AACUE fue negociada sobre la base de un instrumento cargado de mayor rigidez y este hecho conllevó a la existencia de reglas de origen más rígidas en él, lo que representará un verdadero problema para los exportadores costarricenses cuando deban someterse a normas de origen más rígidas para obtener los beneficios del AACUE ya que tras su llegada, Costa Rica será retirada de la lista de beneficiarios del SPG. 
Estos datos fueron comprobados a través de un análisis comparativo de las reglas de origen consagradas en cada Acuerdo. Se analizaron un total de 662 reglas de origen de las cuales el AACUE presentó 257 reglas más rígidas (38,8\%), en tanto que tan solo 66 reglas fueron más flexibles $(9,9 \%)$. Este dato resulta bastante alarmador, ya que, por una parte, los países que permanezcan en las listas del SPG y el SPG+ tendrán mejores condiciones de acceso al mercado europeo que las que tendrá Costa Rica vía AACUE y, por otra, resulta inconcebible que Costa Rica pierda los privilegios anteriormente otorgados a través del $\mathrm{SPG}+$ en lo que refiere a normativa de origen, ya que la presencia de un nuevo acuerdo preferencial debería garantizar mayores privilegios y, de ninguna manera, dejar escapar los que se tenían. Por tanto, este hecho figura como un desliz por parte de los creadores de política comercial costarricenses y de Centroamérica en general.

Tanto Costa Rica como los demás países centroamericanos se encuentran en un periodo de transición que concluirá en su exclusión de la lista de beneficiarios del SPG en un corto plazo. Por este motivo, los demás países centroamericanos enfrentarán la misma situación de Costa Rica una vez que no puedan seguir exportando al margen del SPG.

Adicionalmente fueron analizados los principales 14 productos de exportación costarricenses a la Unión Europea para el año 2013, ya que, al ser sectores competitivos y de interés exportable, conviene, en algunos de los casos, tener reglas de origen más flexibles que faciliten la entrada al mercado europeo. Sin embargo, el AACUE posee reglas más rígidas para 7 de los productos mencionados y ninguna de las reglas es más flexible para estos productos; mientras que, al menos dos de ellos, no poseen cambios entre un régimen y el otro; las restantes no pudieron ser definidas, debido a que obedecen a criterios propios de producción de cada exportador específico.

La migración al AACUE podría lesionar los intereses de 209 empresas costarricenses que durante el año 2014 seguían exportando a la Unión Europea, amparadas al Sistema de Preferencias Generalizadas y que solicitaron un total 5494 certificados de origen para ese año. Si el AACUE mejorara sustancialmente las condiciones de acceso de las mercancías costarricenses al mercado de la UE, consolidando y mejorando las preferencias unilaterales del SGP, sería un motivo para que todos los empresarios quieran migrar al nuevo régimen, pero esto no ocurre.

Si se tiene en cuenta que en Costa Rica el 77\% del parque empresarial en el año 2013 se encontraba representado por PYMES, y de ellas un 74\% eran microempresas en las que se emplea un máximo de 15 personas y, en la mayoría de este tipo de empresas, los recursos no son suficientes para contratar personal especializado en comercio internacional o aduanas; siendo las normas de origen un 
tema tan complejo y lleno de tecnicismos, muchos de estos exportadores serán conscientes de la disyuntiva analizada en la presente investigación hasta el momento en que Costa Rica deje de ser beneficiario del SPG.

Por lo tanto, no cabe duda de que el presente estudio y sus hallazgos permitieron comprobar que tras la disyuntiva en cuanto a tiempo y normativa base para la negociación del AACUE, existen reglas de origen más rígidas en el AACUE en relación con las establecidas en el SPG.

Como conclusión general, es preciso indicar que resulta fundamental que los exportadores costarricenses tengan derecho a la coexistencia entre el régimen de acceso a mercados del SPG (reglamento actual) y el del AACUE, incluyendo la coexistencia del régimen de origen de ambos esquemas preferenciales.

La coexistencia garantizaría que no haya pérdida de preferencias arancelarias al migrar de un sistema (SPG+) a otro sistema (AACUE), especialmente por motivos de incumplimiento de las reglas de origen; de no aplicarse la coexistencia, los países que permanezcan en los listas del SPG podrían tener mejores condiciones de acceso al mercado europeo que las que tendría Costa Rica. 


\section{Referencias}

Acuerdo de Asociación entre Centroamérica y la Unión Europea. (2012B). Relativo a la definición de "Productos originarios" y métodos de cooperación administrativa (Anexo II). Ministerio de Comercio Exterior de Costa Rica. Recuperado de http://goo.gl/fSntrx

Acuerdo General de Aranceles y Comercio. (1947). Acuerdo General de Aranceles y Comercio . Organización Mundial del Comercio. Recuperado de https:// goo.gl/wMw3G2

Brenton, P., \& Manchin, M. (Marzo de 2002). Making EU Trade Agreements Work: The Role of Rules of Origin. Center for European Policy Studies. Recuperado de http://goo.gl/VUYZL0

Castro, A. (5 de marzo de 2015). Conclusión del sistema generalizado de preferencias plus (SGP+) y transición al acuerdo de asociación entre centroamérica y la unión europea (aacue). recuperado el 19 de mayo de 2015, de ministerio de comercio exterior de costa rica: http://goo.gl/wmbkkd

Céspedez vargas, r. (octubre de 2009). negociaciones del acuerdo de asociacion centroamérica-unión europea, retos y oportunidades para los sectores productivos de los países centroamericanos. recuperado el 03 de mayo de 2015, de fundación friedrich ebert: http://goo.gl/ijufcs

Comisión de las comunidades europeas. (18 de diciembre de 2003). libro verde: el futuro de las normas de origen en los regímenes comerciales preferenciales. recuperado el 15 de abril de 2015, de eur-lex: http://goo.gl/alxmnv

Comisión de las comunidades europeas. (16 de marzo de 2005). comunicación de la comisión al consejo, al parlamento europeo y al comité económico y social: las normas de origen en los regímenes comerciales preferenciales, orientaciones de cara al futuro. EUR-Lex. Recuperado de http://goo.gl/BYlWgK

Comisión Europea. (Julio de 2005). Normas de Origen del Sistema de Preferencias Generalizadas de la Comunidad Europea. Ministerio de Comercio Exterior y Turismo de Perú. Recuperado de http://goo.gl/LJlZRG

Comisión Europea. (09 de enero de 2004). Notificación relativa al Libro Verde de la Comisión «El futuro de las normas de origen en los regímenes comerciales preferenciales». EUR-Lex. Recuperado de http://goo.gl/39mU6P

Comisión Europea. (22 de julio de 2014). Reglamento Delegado UE N. ${ }^{\circ}$ 1015/2014 . Boletín Oficial del Estado, BOE. Recuperado de http://goo.gl/M4uSlK

Consejo de la Unión Euopea. (06 de agosto de 2008). Reglamento (CE) N. ${ }^{\circ}$ 732/2008 . EUR-Lex. Recuperado de http://goo.gl/jUE2Fp 
Consejo de la Unión Europea. (18 de noviembre de 2010). Reglamento (UE) $N .^{\circ}$ 1063/2010. EUR-Lex. Recuperado de http://goo.gl/3Mwym9

Corrales, M. (15 de noviembre de 2011). Autoridad Certificadora de Origen. Promotora de Comercio Exterior de Costa Rica. Recuperado de http://goo.gl/voldXS

Corrales, M. (25 de mayo de 2012). El Régimen de Origen en los Tratados de Libre Comercio. (C. Cordero Madrigal, Entrevistador).

Corrales, M. (2013). Proceso de Certificación Acuerdo de Asociación entre Centroamérica y la Unión Europea. Promotora de Comercio Exterior de Costa Rica, PROCOMER. Recuperado de http://goo.gl/pBICH4

Dirección General de Comercio de la Comisión Europea. (Marzo de 2004). Sistema de Preferencias Generalizadas de la Unión Europea. Comisión Europea. Recuperado de http://goo.gl/qNvP0K

Estevadeordal, A. (Mayo de 2003). El impacto de los acuerdos comerciales preferenciales sobre el comercio de bienes. Banco Interamericano de Desarrollo. Recuperado de http://goo.gl/K7EX91

Etevadeordal, A., \& Suominen, K. (2005). Rules of Origin in Preferential Trading Arrangements: Is All Well with the Spaghetti Bowl in the Americas? Research Gate. Recuperado de http://goo.gl/P64avb

Govaere, V. (2011). Opotunidades y desafíos del Acuerdo de Asociación con la Unión Europea. Portal de Revistas Académicas de la Universidad de Costa Rica. Recuperado de http://goo.gl/R0ct0r

Lamy, P. (20 de junio de 2011). Iniciativa Hecho en todo el mundo. MéxicoXport. Recuperado de http://goo.gl/D6asXY

López Libreros, J. M. (2008). Las Normas de Origen para las Mercancías en el Sistema GATT-OMC. Revista de Información Comercial Española, ICE. Recuperado de http://goo.gl/UtsRpP

Martínez Jerez, A. M. (2011). Aprobación del Nuevo Reglamento de Normas de Origen en el Marco del Sistema de Preferencias Generalizadas. Revista de Información Comercial Española, ICE. Recuperado de http://goo.gl/Pytjyz

Mata, H., \& Cordero, M. (Octubre de 2009). El Acuerdo de Asociación Económica entre Centroamérica y la Unión Europea: Viabilidad, avances y perspectivas. Comisión Económica Para América Latina, CEPAL. Recuperado de http://goo.gl/45y6Lt

Ministerio de Comercio Exterior de Costa Rica. (2012). Acuerdo de Asociación entre Centroamérica y la Unión Europea. Documento Explicativo (2da Ed.). San José, Costa Rica: COMEX. 
Ministerio de Economía y Competitividad de España. (2015). Sistema de Preferencias Generalizadas. Ministerio de Economía y Competitividad de España. Recuperado de http://goo.gl/TCE67d

OMC E-Learning. (Febrero de 2014). Los Acuerdos Multilaterales Sobre el Comercio en la OMC. OMC. Recuperado de https://goo.gl/KK9BTU

Organización Mundial del Comercio. (1994). Acuerdo Sobre Normas de Origen. OMC. Recuperado de https://goo.gl/7RIufe

Organización Mundial del Comercio. (Mayo de 2015A). Acuerdos comerciales regionales y arreglos comerciales preferenciales. OMC. Recuperado de https://goo.gl/xrokax

Organización Mundial del Comercio. (Mayo de 2015B). Algunas cifras sobre los acuerdos comerciales regionales notificados ante el GATT / la OMC y en vigor. OMC. Recuperado de http://goo.gl/FRewYb

Organización Mundial del Comercio. (2015D). Información técnica sobre las normas de origen. OMC. Recuperado de https://goo.gl/qrKXax

Organización Mundial del Comercio. (2007). Informe sobre el comercio mundial, seis decenios de cooperación comercial multilateral. OMC. Recuperado de https://goo.gl/rUSI5d

Organización Mundial del Comercio. (2015E). Las Normas de Origen. OMC. Recuperado de https://goo.gl/qmIHUV

Pérez, R., \& Grether, J. (Septiembre de 1998). Flujos de comercio preferencial y no preferencial en el comercio mundial. Organización Mundial de Comercio. Recuperado de https://goo.gl/EuRCBn

Piñuel Raigada, J. L. (2002). Epistemología, metodología y técnicas del análisis de contenidos. Universidad Complutense de Madrid, UCM. Recuperado de https://goo.gl/OwyJPr

Servicio Europeo de Acción Exterior. (2015). Relaciones de la UE con Costa Rica. Servicio Europeo de Acción Exterior. Recuperado de http://goo.gl/a5kcCK 